\title{
Incomplete information and incentives to free ride
}

\author{
Mehmet Bac \\ Bilkent University, Department of Economics, Bilkent, Ankara 06533, Turkey
}

Received: 18 August 1994/Accepted: 15 June 1995

\begin{abstract}
We study private provision of a continuous public good in an incomplete information repeated game. The analysis generates the following predictions in the form of Perfect Bayesian equilibria. When the discount factor is low, the game played by increasingly optimistic players may collapse into a war of attrition: delay may occur and one player may bear the burden of providing the public good while the other free rides forever. On the other hand, if the discount factor is high, or low but prior beliefs are "pessimistic", the inclusion of incomplete information has no impact on the pattern of contributions.
\end{abstract}

\section{Introduction}

The contributor to any public good obtains a direct benefit from the increased supply, but defectors with the same valuation as the contributor usually reap a higher net benefit. This externality leads to the well-known underprovision result obtained in static, noncooperative models. However, the theory predicts quite the opposite for the case of recurring contributions: Under complete information, McMillan (1979) has shown that the repeated public good provision game has equilibria on the payoff possibility frontier. The purpose of this paper is to investigate the free-riding problem in a dynamic model with incomplete information about valuations, and contrast the resulting pattern of contributions with the polar predictions mentioned above.

We consider a repeated provision game of a continuous and perishable public good. In each stage game the players' welfares depend on total contributions raised during the corresponding period. Real world situations involving such public goods are, for example, countries sharing a global ecosystem, students sharing facilities of a dormitory, partnership games (analyzed by Radner 1986) and the interaction of two superpowers in a small country (Schelling 1966, Ch. 2). The common features of these situations are the presence of positive externalities and 
individual actions that are strategic substitutes. ${ }^{1}$ Whereas equilibria of static games display free riding, repeated play of these games generates incentives to cooperate and obtain higher payoffs through the possibility of retaliation. The inclusion of incomplete information complicates the problem, however. If a pure defection advantage exists, a high-valuation player faces a dilemma: by making a contribution he reveals his type, hence his threats to defect in the succeeding periods will lack credibility. On the other hand, if everyone defects nothing gets done and all high-valuation players are worse off. This brings us to central questions addressed in this paper: Under what conditions does a pure defection advantage arise? How is the pattern of contributions affected by the inclusion of incomplete information in a repeated game?

Three types of inefficiencies are emphasized in the literature on private provision of public goods. The first is the well known free-rider problem, mostly observed in static contexts. ${ }^{2}$ The second inefficiency manifests itself in the form of delayed contributions in dynamic settings with incomplete information. ${ }^{3} \mathrm{~A}$ third type of inefficiency also arises in dynamic models, generating total contributions even lower than predicted by static models. Such an equilibrium outcome involves either a small number of contributors or many contributors with very low individual contributions (see Fershtman and Nitzan 1991 or Admati and Perry 1991). These three types of inefficiencies are all potentially present in our model. The main improvement of this paper to the existing literature lies in its rich class of predictions regarding the link between the initial parameters (i.e., distribution of prior beliefs, the types and the discount factor of the players) and the properties of potential outcomes (i.e., type of inefficiency, the pattern of contributions, identities of the contributors, occurrence of delay before the first contribution). This stems from the "nature" of our stage game. For instance, if the stage game has the features of a chicken game, its repeated play under incomplete information yields a war of attrition, whereas an efficient outcome with high contributions may arise if the stage game has the features of a prisoners' dilemma. To gain insight into the question of when the provision game generates a specific type of inefficiency (such as those observed in a war of attrition), the stage game of the overall game wherein the potential contributors' incentives are analyzed must be sufficiently flexible. Our model has this property; for some values of the parameters the stage game turns

\footnotetext{
${ }^{1}$ Individual actions are strategic substitutes if best-reply functions have negative slopes. In the context of a public good provision problem, strategic substitutability implies that a player's optimal reaction to an increase in other players' contributions is to decrease his own contribution. See Fudenberg and Tirole (1984) or Bulow et al. (1985).

${ }^{2}$ See for example Palfrey and Rosenthal (1984, 1988), Bergstrom et al. (1986) and Andreoni (1988) for recent treatments of the classical free-rider problem in static contexts.

${ }^{3}$ The analysis of this outcome is closely related to the war of attrition games (see, e.g., Nalebuff and Riley (1985) and Fudenberg and Tirole (1986), bargaining under incomplete information (Chatterjee and Samuelson 1987) and reputation (Kreps and Wilson 1982). The first paper to introduce dynamics and delay in a public good provision context is Bliss and Nalebuff (1984) but their analysis is limited to a discrete public good and the game terminates at the provision date. Gradstein (1992) has extended their model to two periods with accumulating contributions. In his model, individual contributions and provision costs are fixed but costs vary across individuals. This paper is most closely linked to the last two papers mentioned above. Significant differences between these models and ours are presented in Section 2.
} 
into a chicken game, for some others, into a prisoners' dilemma. As expected, total contributions under these two types of stage games evolve quite differently.

The results and the contents of the paper are summarized below. We present in Section 2 a two-player repeated game with two potential types for each player. While the low-valuation player never contributes to the public good, the highvaluation type may contribute unilaterally. One of the factors on which the pattern of contributions in the overall game depends is the expected outcome of the potential subgame played under complete information. Here we make the standard assumption that in this subgame the high-valuation types play a "focal equilibrium" with symmetric and efficient payoffs subject to the subgame-perfection constraint. These payoffs, hence the Perfect Bayesian equilibrium of the overall game, depend on the discount factor. In Section 4 we characterize these equilibria and show that for discount factors not too low, the inclusion of incomplete information is inconsequential to the pattern of contributions. This result is in the spirit of the folk theorem and highlights the conditions under which two highvaluation players will provide the public good cooperatively forever no matter their beliefs at the outset of the game.

Another potential outcome of the overall game is a war of attrition. In Section 4 we show that the necessary condition for this outcome is a low discount factor. However, a low discount factor is not sufficient; If the players put much weight on their actual welfares or if they think that the situation will not recur any more, then the outcome depends critically on the distribution of prior beliefs. For instance, the war of attrition is avoided if all players are pessimistic, i.e., if each thinks he is the only player with a high valuation. Otherwise the inclusion of incomplete information leads to a war of attrition where the outcome may exhibit all three types of inefficiencies mentioned earlier. In a war of attrition, strategies are mixed at the initial stages, hence convey type-information and one player (or both) may reveal his type in any period, converting the overall game into an asymmetric (or complete) information game. We show that more optimistic prior beliefs can actually intensify the players' incentives to free ride even in a repeated game setting. If prior beliefs are asymmetric and the discount factor is low, the war of attrition is resolved immediately: With probability one, the pessimistic player starts contributing and bears the burden of providing the public good while the other player free rides forever.

Section 5 concludes.

\section{The model}

This section presents a two-player, infinite horizon repeated game of private provision of a continuous public good, $Q$. We consider two basic types of players. A type- $L$ player never participates. The other type, called type- $H$, has a sufficiently high valuation so that he may participate. Denoting by $C(Q)$ and $U(Q)$ respectively the per-period provision cost and the utility function of a type- $H$ player, we assume the following:

(A1) $U(Q)$ is strictly concave, increasing and $C(Q)$ is strictly convex and increasing. Furthermore, $U(0)=C(0)=0$ and $\lim _{Q \rightarrow 0} U^{\prime}(Q)>\lim _{Q \rightarrow 0} C^{\prime}(Q)>0$.

According to (A1), there is a range of $Q$ in which the marginal utility of the type- $H$ player exceeds his marginal cost. This simple structure can be deduced from a more elaborate model including private goods. The opportunity cost of providing the 


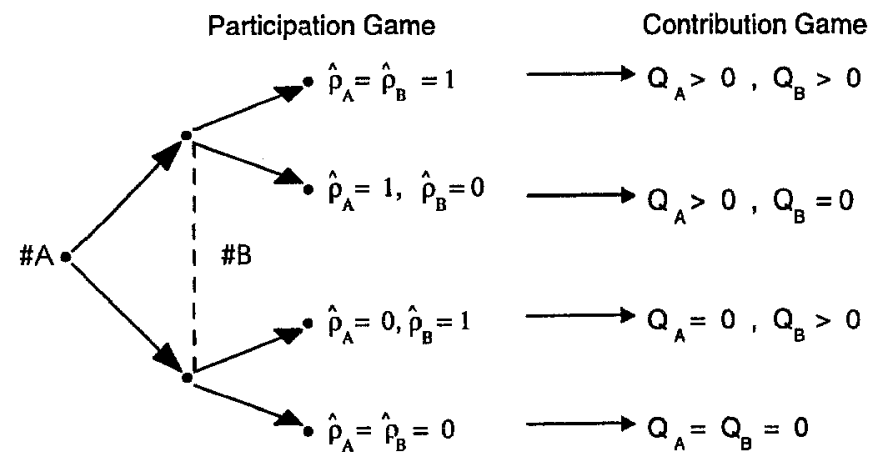

Fig. 1

public good would then be measured in terms of the private good. The two players, $A$ and $B$, have the same discount factor, $0<\delta<1$. It is common knowledge that player $i$ is of type $H$ with probability $\pi_{i}$ but types are privately known.

We decompose the action sets of the players in the stage game into two sequential moves (see Fig. 1): First, they decide simultaneously on whether to contribute (participation strategy). Next, the players who have decided to contribute determine the size of their contributions (contribution strategy). The stage game can accordingly be decomposed into one participation game and one contribution game. The outcomes of both participation and contribution games are assumed to be observable. ${ }^{4}$ At the end of each period, total contributions are consumed and the game proceeds to the next period. Since type- $L$ s never contribute anything, a participation with a positive contribution is a clear-cut signal that the contributor is of type- $H$. For $i=A, B$, the range of a mixed participation strategy $\rho_{i}$ is $[0,1]$, and the realization of $\rho_{i}$ is denoted $\hat{\rho}_{i}$. If a player participates $\left(\hat{\rho}_{i}=1\right)$, his contribution strategy $Q_{i}$ may take any value in $R^{+}$. Clearly, $\hat{\rho}_{i}=0$ implies $Q_{i}=0$. Since participation precedes contribution, the domain of $Q_{i}$ consists of possible realizations of the participation strategy.

Let us now compare the features of the game presented above with those of the two most closely linked papers, Bliss and Nalebuff (1984) and Gradstein (1992). First, the stage game in this paper is repeated ad infinitum whereas the provision game of Bliss and Nalebuff terminates at the provision date if and only if at least one player makes a contribution. Gradstein extends their model to two periods by assuming accumulating total contributions. In both papers, each individual has a fixed cost of contributing a fixed amount, thus faces a simple binary decision problem. Second, these are models of discrete, durable public goods, not of

\footnotetext{
${ }^{4}$ The decomposition of the stage game, hence of the strategies of the players, is technically useful because dealing with randomizations over a binary choice set when players hesitate between participation and defection is much easier than studying randomizations over a continuum of contribution strategies. Furthermore, as explained below, the decision to participate (i.e., choosing a positive contribution) is a crucial factor affecting the evolution of the game because it reveals the contributor's type. Treating participation strategies separately highlights this leakage of information.
} 
continuous and perishable public goods as considered here. ${ }^{5}$ Third, some features of the models in these two papers stem from the authors' interest in the asymptotic properties of contribution equilibria, as population size approaches infinity. The model in the present paper takes as given the population size and focuses on recurrent interactions.

We now present formal definitions of the equilibrium concept and strategies in the overall game. Let $\alpha^{t}=\left(\left\{\hat{\rho}_{A}^{t}, Q_{A}^{t}\right\} ;\left\{\hat{\rho}_{B}^{t}, Q_{B}^{t}\right)\right)$ be the actions in period $t$. A history of the play at period $t$ is defined by $h^{t}=\left(\alpha^{1}, \alpha^{2}, \ldots, \alpha^{t-1}\right)$. A mixed participation strategy for player $i$ in the overall game is a sequence of maps $\rho_{i}^{t}$, one for each period $t$, from possible histories $h^{t}$ into $[0,1]$. Similarly, a contribution strategy for the overall game maps $h^{t} \times[0,1]^{2}$ into $R^{+}$, for each $t$. Since these strategies may convey type-related information, at the teginning of each period the players use their opponent's previous participation strategies to update their beliefs according to Bayes' rule in the obvious way. Player $i$ 's belief that Player $-i$ is of type- $H$ in period $t$ is a function $\pi_{-i}^{t}:\{t\} \rightarrow[0,1]$ such that, given $\pi_{-i}^{t-1}, \rho_{i}^{-t-1}$ and $\rho_{-i}^{t-1}$,

$$
\pi_{-i}^{t}=\left\{\begin{array}{cl}
1 & \text { if } \hat{\rho}_{-i}^{s}=1 \text { for any } s<t ; \\
\frac{\pi_{-i}^{t-1}\left(1-\rho_{-i}^{t-1}\right)}{\pi_{-i}^{t-1}\left(1-\rho_{-i}^{t-1}\right)+\left(1-\pi_{-i}^{t-1}\right)} & \text { if } \hat{\rho}_{-i}^{s}=0 \text { for all } s<t .
\end{array}\right.
$$

Beliefs are thus required to be consistent with strategies and satisfy the "never dissuaded once convinced" condition. Note that in the period in which the other player contributes, $\pi_{-i}$ is updated to (and will remain equal to) one. If, on the other hand, no contribution is observed, a slightly complex rule is used along the equilibrium path. Mixed participation strategies (not their realizations) appear in this updating rule; rational players are able to predict accurately their opponents' strategies along the equilibrium path.

Let $W_{A}^{t}$ and $W_{B}^{t}$ denote respectively $A$ 's and $B$ 's expected discounted payoffs as viewed from period $t$. We require the strategies in the overall game to form a Perfect Bayesian equilibrium (PBE), defined below:

A Perfect Bayesian Equilibrium is a pair of strategy sequences $\left\{\rho_{A}^{t^{*}}, Q_{A}^{t^{*}}\right\}$ and $\left\{\rho_{B}^{t^{*}}, Q_{B}^{t^{*}}\right\}$ of type- $H$ players such that, for all $t$, given the history $h^{t}$ and consistent beliefs $\left\{\pi_{A}^{t}\right\}$ and $\left\{\pi_{B}^{t}\right\}$,

$$
\begin{array}{ll}
W_{A}^{t}\left(\left\{\rho_{A}^{t^{*}}, Q_{A}^{t^{*}}\right\},\left\{\rho_{B}^{t^{*}}, Q_{B}^{t^{*}}\right\}\right) \geq W_{A}^{t}\left(\left\{\rho_{A}^{t}, Q_{A}^{t}\right\},\left\{\rho_{B}^{t^{*}}, Q_{B}^{t^{*}}\right\}\right) & \text { for all }\left\{\rho_{A}^{t}, Q_{A}^{t}\right\} \\
W_{B}^{t}\left(\left\{\rho_{A}^{t^{*}}, Q_{A}^{t^{*}}\right\},\left\{\rho_{B}^{t^{*}}, Q_{B}^{t^{*}}\right\}\right) \geq W_{B}^{t}\left(\left\{\rho_{A}^{t^{*}}, Q_{A}^{t^{*}}\right\},\left\{\rho_{B}^{t}, Q_{B}^{t}\right\}\right) \text { for all }\left\{\rho_{B}^{t}, Q_{B}^{t}\right\} .
\end{array}
$$

\footnotetext{
${ }^{5}$ Bliss and Nalebuff's model is mainly aimed at explaining delay in contributions. Although their model is dynamic, it ignores the implication of providing the public good by assuming away the possibility that the same players may face again the same situation. Clearly, the player who has provided the public good in the first round will provide it in the second round as well. If so, what happens to the outcome of the first round? Gradstein's extension provides a partial answer to this question in a model that combines Bliss and Nalebuff's paper with Fershtman and Nitzan (1991): Players are more reluctant to participate in the two-period case than in the one-period case. We show that this prediction may change dramatically when the game is repeated ad infinitum; the inclusion of incomplete information may not produce further inefficiencies in the supply of public goods.
} 
The PBE concept basically requires that the strategies be sequentially rational (optimal given beliefs and the history of the game) and that beliefs be updated according to Bayes' rule whenever possible.

\section{Analysis of the potential subgames}

We focus in this section on the stage game and some special subgames of the overall game, and study their equilibria. The results will be used later in Section 4 . The subgames considered here have special information structures which correspond to $\pi_{i}^{1} \in\{0,1\}$, that is, complete information about types. Since the case of two type- $L$ players $\left(\pi_{i}^{1}=0\right)$ is uninteresting, we consider the remaining three combinations of prior beliefs and analyze the corresponding repeated game (or any of its subgames).

When it is common knowledge that one player is of type- $H$ while the other is of type- $L$, the game has a trivial solution: The type- $H$ player produces the public good forever with unilateral contributions $Q^{\mathrm{HL}}=\operatorname{argmax}_{Q}\{U(Q)-C(Q)\}$, which is unique by (A1). The overall game has subgames in which the equilibrium outcome exhibits perpetual unilateral contributions; these subgames are called the CN-game or the NC-game ( $\mathrm{C}$ for contributing, $\mathrm{N}$ for not contributing), depending on whether it is player $A$ or $B$ who unilaterally provides the public good forever. Finally, the overall game played by two type- $H$ players under complete information $\left(\pi_{i}^{1}=1\right)$, or any subgame of the overall game having this information structure is called the CC-game. We will analyze this game below in some detail.

Suppose, then, that $\pi_{i}=1$ is common knowledge. Player $i$ 's objective in the static stage game is

$$
\max _{\left\{\rho_{i}, Q_{i}\right\}}\left[\rho_{i}\left\{\left[\rho_{j} U\left(Q_{i}+Q_{j}\right)+\left(1-\rho_{j}\right) U\left(Q_{i}\right)\right]-C\left(Q_{i}\right)\right\}+\left(1-\rho_{i}\right) \rho_{j} U\left(Q_{j}\right)\right]
$$

Since, ex-ante, player $j$ 's strategies are unknown to player $i, \rho_{j}$ can be interpreted as the probability that $j$ will participate. By (A1) individual contributions are strategic substitutes, i.e., $d Q_{i}^{*} / d Q_{j}^{*}<0$ : the optimal contribution of player $i$ decreases as player $j$ increases his contribution. Because the stage game is strongly symmetric, it has a unique symmetric Nash equilibrium in which each player participates and contributes $Q^{\mathrm{HH}}=\operatorname{argmax}_{Q}\left\{U\left(Q+Q^{\mathrm{HH}}\right)-C(Q)\right\}$. The low payoffs corresponding to symmetric contributions $Q^{\mathrm{HH}}$, denoted $\underline{P}$ in Fig. 2, represent the first type of

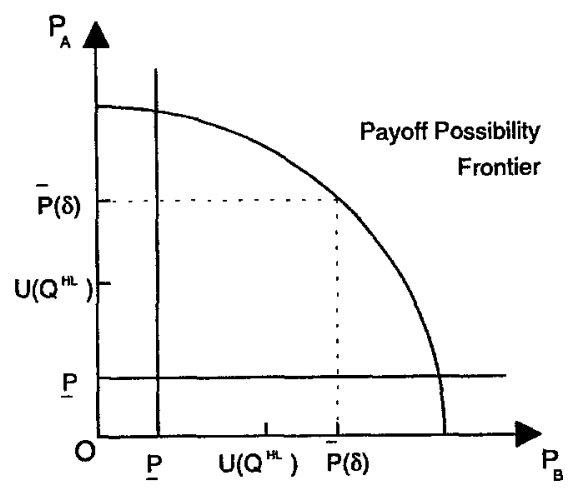

Fig. 2 
inefficiency observed in static settings. As a reference point, it is useful to determine the efficient supply of public good with symmetric contributions, denoted $Q^{F}$. These solve the following problem:

$$
\max _{\left\{Q_{1}, Q_{2}\right\}}\left[2 U\left(Q_{1}+Q_{2}\right)-C\left(Q_{1}\right)-C\left(Q_{2}\right)\right] .
$$

From the corresponding first-order condition $2 U^{\prime}\left(2 Q^{\mathrm{F}}\right)=C^{\prime}\left(Q^{\mathrm{F}}\right)$, it follows that $Q^{\mathrm{F}}>Q^{\mathrm{HH}}$. We denote by $\bar{P}$ the symmetric payoff $U\left(2 Q^{\mathrm{F}}\right)-C\left(Q^{\mathrm{F}}\right)$. Thus the lower and upper bounds of feasible symmetric total payoffs for the static stage game are respectively $2 \underline{P}$ and $2 \vec{P}$.

We consider now the CC-game, the infinitely repeated stage game under complete information. This game has subgame perfect equilibria (SPE) in which type- $H$ players reach higher payoffs via the penalty of Nash reversion. From the well-known perfect folk theorem, any individually rational and feasible average payoff (up to the first-best) can be supported by SPE strategies provided that $\delta$ is sufficiently high. Here this first-best outcome corresponds to total payoffs as large as $2 \bar{P}$.

There is no agreed-upon way of choosing from the set of SPE of the CC-game; most authors focus exclusively on an efficient equilibrium, usually a symmetric one, yet some versions of the "renegotiation proofness" concept used by a number of authors imply that the equilibrium must be inefficient. Here we adopt the former approach widely used (implicitly or explicitly) in the literature. Denoting by $\{\bar{P}(\delta), \bar{P}(\delta)\}$ the highest symmetric pair of payoffs that can be supported as a SPE outcome given the discount factor, we thus make the following assumption:

(A2) The players in the CC-game play their symmetric and most efficient SPE strategies and attain the payoffs $\bar{P}(\delta)$.

That is, each player in the CC-game contributes $\bar{Q}(\delta)$ maximizing the discounted payoff $[U(2 Q)-C(Q)] /(1-\delta)$ subject to

$$
\bar{P}(\delta)=(1-\delta)\left[U\left(Q^{*}(\bar{Q})+\bar{Q}(\delta)\right)-C\left(Q^{*}(\bar{Q})\right)\right]+\delta \underline{P} .
$$

(4) is called the subgame-perfection constraint, where $Q^{*}(\bar{Q})$ is the static best reply to $\bar{Q}(\delta) .{ }^{6} \mathrm{~A}$ deviation to the best static reply yields in the actual period the payoff $U\left(Q^{*}(\bar{Q})+\bar{Q}(\delta)\right)-C\left(Q^{*}(\bar{Q})\right)$, however the path of contributions then reverts to the static Nash equilibrium where individual payoffs are $\underline{P}$. The maximum symmetric payoff pair is a point on the payoff possibility frontier (see Fig. 2), which shrinks as $\delta$ decreases. If (4) holds, no player will deviate from the per-period contribution $\bar{Q}(\delta)$, hence each player will obtain the per-period payoff $\bar{P}(\delta)$. A first implication of (A2) on the equilibria of the overall game is stated below.

Proposition 1. If $\underline{P} \geq U\left(Q^{\mathrm{HL}}\right)$, the incomplete information overall game switches immediately to the CC-game for any priors and discount factor.

\footnotetext{
${ }^{6}$ The argument in favor of the players' being attracted by the "focal point" described in (A2) is the "equal-sharing principle", a very influential force in social contexts as the public good provision game analyzed here. Also, the payoff functions of two type- $H$ players are perfectly symmetric. Furthermore, among the symmetric equilibria, all players would prefer the one with highest attainable payoff $\bar{P}(\delta)$, given the discount factor that sustains this outcome as a SPE. There is strong evidence for the power of focal points in determining outcomes, even in isolated bargaining experiments where forces favoring self-interested behavior are the most effective. See Roth (1987) for a review of experiments that illustrate this phenomenon.
} 
Proof. $\underline{P}$ is the lowest attainable symmetric SPE payoff in the CC-game, while $U\left(Q^{\mathrm{HL}}\right)$ is the payoff corresponding to a pure defection strategy when the other player makes his optimal unilateral contribution $Q^{\mathrm{HL}}$. Thus if $\underline{P} \geq U\left(Q^{\mathrm{HL}}\right)$, there is no incentive to conceal type-information; both players participate in the first period to reap higher payoffs of the CC-game. Q.E.D.

In addition to (A1) and (A2), throughout the rest of the paper we assume:

(A3) $\underline{P}<U\left(Q^{\mathrm{HL}}\right)$.

That is, the condition in Proposition 1 does not hold. The case represented in Fig. 2 corresponds to (A3). The stage game then becomes a chicken game: the Nash mutual participation payoff $\underline{P}$ is better than mutual defection, but each player's most preferred outcome is that he defects while the other player contributes. As we show in Section 4, (A3) is also necessary but not sufficient for a free-riding advantage to arise from the information problem alone.

The last subgame we consider is the $\mathrm{CN}$-game where $B$ is the informed player (corresponding prior beliefs are $\pi_{A}^{1}=1$ and $\pi_{B}^{1} \in[0,1)$ ). This game has a unique undominated PBE: If the discount factor is sufficiently high so that $P(\delta) \geq U\left(Q^{\mathrm{HL}}\right)$, then the $\mathrm{CN}$-game collapses immediately to the CC-game. Otherwise, if $\bar{P}(\delta)<U\left(Q^{\mathrm{HL}}\right)$, the informed player prefers concealing his unverifiable type and chooses a perpetual defection strategy. ${ }^{7}$ The uninformed player's threat "I will stop contributing if you (the informed player) do not contribute as well" is empty, because there is a probability (however small) that the informed player is of type- $L$. Hence under these conditions the uninformed player is bound to provide the public good forever.

\section{Equilibria of the overall game}

Since the discount factor is an important determinant of equilibrium contribution patterns, we provide below its possible interpretations before proceeding with the formal analysis. The variable $\delta$ is usually thought of as representing pure time preference, that is, $\delta=e^{-r \Delta}$ where $\Delta$ is the length of each period and $r$ is the rate of time preference. $\delta$ decreases as $r$ and/or $\Delta$ increases. A higher length of period would mean a lower frequency of interaction between players. Another possible interpretation of $\delta$ is the probability of continuing from one period to the next, or the probability that the same public good provision problem will manifest itself again.

Consider now the outset of the overall game with prior beliefs $\pi_{A}^{1}$ and $\pi_{B}^{1}$. The first step of the analysis is to determine the conditions under which incomplete valuation information generates a pure defection advantage. We start with the following proposition:

Proposition 2. The strategy configuration $\left\{\rho_{A}\right\}=\left\{\rho_{B}\right\}=\{0,0, \ldots\}$ is not a PBE of the overall game played by type-H players.

That is, mutual perpetual defection through pure strategies is not an equilibrium outcome if at least one player is of type $H$. The proof is straightforward and is

\footnotetext{
${ }^{7}$ The $\mathrm{CN}$ - and NC-game are special potential subgames of the overall game, whose equilibria are characterized in Proposition 3. The proof of Proposition 3 can thus easily be adapted to the PBE of the CN- or NC-game.
} 
omitted. Discounted payoffs of a type- $H$ player associated with these strategies are zero, thus by (A1) a deviation in any period enhances welfare. As we show in Proposition 4 below, the overall game must eventually reach one of the three potential subgames among which the $\mathrm{CN}$ and NC-game exhibit perpetual unilateral defections. Now, the expected discounted payoffs of $A$ and $B$ can respectively be written as: ${ }^{8}$

$$
\begin{aligned}
W_{A}^{1}= & \rho_{A}^{1}\left[\rho_{B}^{1} \pi_{B}^{1} W_{A}^{\mathrm{CC}}+\left(1-\rho_{B}^{1} \pi_{B}^{1}\right) W_{A}^{\mathrm{CN}}\right]+\left(1-\rho_{A}^{1}\right)\left[\rho_{B}^{1} \pi_{B}^{1} W_{A}^{\mathrm{NC}}\right. \\
& \left.+\left(1-\rho_{B}^{1} \pi_{B}^{1}\right) \delta W_{A}^{2}\right], \\
W_{B}^{1}= & \rho_{B}^{1}\left[\rho_{A}^{1} \pi_{A}^{1} W_{B}^{\mathrm{CC}}+\left(1-\rho_{A}^{1} \pi_{A}^{1}\right) W_{B}^{\mathrm{NC}}\right]+\left(1-\rho_{B}^{1}\right)\left[\rho_{A}^{1} \pi_{A}^{1} W_{B}^{\mathrm{CN}}\right. \\
& \left.+\left(1-\rho_{A}^{1} \pi_{A}^{1}\right) \delta W_{B}^{2}\right] .
\end{aligned}
$$

In (5), the squared bracket following $\rho_{A}^{1}$ represents player $A$ 's expected discounted payoff from participating, and the one following $\left(1-\rho_{A}^{1}\right)$, from defecting in period one. In the superscripts of $W_{A}$, combinations of $C$ and $N$ denote player $A$ 's expected discounted payoff from the three potential subgames $\mathrm{CC}, \mathrm{CN}$ and $\mathrm{NC}$. For instance, if the outcome of the participation game in period one is $\hat{\rho}_{i}^{1}=1$ for $i=A, B$, then the game collapses into the complete information CC-game where average (per-period) payoffs are $\bar{P}(\delta)$. Thus

$$
W_{A}^{\mathrm{CC}}=W_{B}^{\mathrm{CC}}=\frac{\bar{P}(\delta)}{1-\delta}
$$

denotes the discounted payoffs if both players supply the public good immediately in period one. This is a random event when mixed participation strategies are used (we have omitted the arguments of the strategies for conciseness). The probability of switching to the CC-game is $\rho_{A}^{1} \rho_{B}^{1} \pi_{B}^{1}$ from the perspective of $A$, and $\rho_{B}^{1} \rho_{A}^{1} \pi_{A}^{1}$ from the perspective of $B$. If $A$ participates and observes no participation from $B$, the game collapses into the $\mathrm{CN}$-game where $B$ defects indefinitely. Then, $A$ 's discounted payoffs are:

$$
W_{A}^{\mathrm{CN}}=\frac{\left[U\left(Q^{\mathrm{HL}}\right)-C\left(Q^{\mathrm{HL}}\right)\right]}{1-\delta} .
$$

Analogously, the outcome in which $A$ becomes the free rider yields

$$
W_{A}^{\mathrm{NC}}=\frac{U\left(Q^{\mathrm{HL}}\right)}{1-\delta} \text {. }
$$

By symmetry, $W_{A}^{\mathrm{CN}}=W_{B}^{\mathrm{NC}}$ and $W_{A}^{\mathrm{NC}}=W_{B}^{\mathrm{CN}}$. Note that revealing a high valuation after the other player has done so is dominated by joint switching to the CC-game in the previous period, because the latter strategy configuration avoids discounting. Finally, if no contribution occurs the game proceeds one period ahead. $A$ 's expected discounted payoffs are $\delta W_{A}^{2}$ and $B$ 's are $\delta W_{B}^{2}$. We can now proceed to characterize the PBE of the overall game, starting with equilibria in pure participation strategies. Let us define $\delta_{c}$, a critical value of the discount factor by

$$
\bar{P}\left(\delta_{c}\right)=U\left(Q^{\mathrm{HL}}\right) .
$$

\footnotetext{
${ }^{8}$ Because the strategy of the type $L$ is known, we need only present the strategies and payoffs of the type $H$ when defining and analyzing equilibria. This, of course, does not imply that the players in the game are known to be of type $H$.
} 
The highest symmetric payoff that can be supported as a SPE outcome in the CC-game is just equal to the pure defection payoff $U\left(Q^{\mathrm{HL}}\right)$ if $\delta=\delta_{c}$. (A3) ensures the existence of $\delta_{c} \in(0,1)$.

Proposition 3: (i) Assume $\delta \geq \delta_{c}$. Play in the overall game switches immediately to the CC-game where the $P B E$ strategies are: $\left\{\rho_{i}^{* 1}\right\}=\{1,1,1, \ldots\}$, $\left\{Q_{i}^{1 *}\right\}=\{\bar{Q}(\delta), \bar{Q}(\delta), \ldots\}$ and beliefs form the sequence $\left\{\pi_{i}^{1}\right\}=\left\{\pi_{i}^{1}, 1,1, \ldots\right\}$ for $i=A, B$. Out-of-equilibrium-path behavior is as follows: If player $i$ deviates to $\rho_{i}^{1}=0$ in period one, player $j$ contributes the best reply $Q^{\mathrm{HL}}$ until $\rho_{i}=1$ is observed. If a deviation occurs in period $t>2$, then the static equilibrium with contributions $Q^{\mathrm{HH}}$ is played forever.

(ii) Assume $\delta<\delta_{c}$. There is a critical region of prior beliefs defined by the function $\bar{\pi}:\left[0, \delta_{c}\right] \rightarrow[0,1]$, such that, if $\pi_{i}^{1} \leq \bar{\pi}(\delta)$, the equilibrium is as described in case (i) above. On the other hand, if $\pi_{i}^{1} \leq \bar{\pi}(\delta)<\pi_{j}^{1}$, then the following strategies constitute the unique PBE:

Player $i:\left\{\rho_{i}^{* 1}\right\}=\left(Q_{i}^{1 *}\right\}=\{0,0, \ldots\}$,

Player $j:\left\{\rho_{j}^{* 1}\right\}=\{1,1, \ldots\},\left\{Q_{j}^{1 *}\right\}=\left\{Q^{\mathrm{HL}}, Q^{\mathrm{HL}}, \ldots\right\}$,

with corresponding belief sequences given by $\left\{\pi_{i}\right\}=\left\{\pi_{i}^{1}, \pi_{i}^{1}, \ldots\right\}$ and $\left\{\pi_{j}\right\}=$ $\left\{\pi_{j}^{1}, 1,1, \ldots\right\}$. Player $j$ 's deviations are ignored, whereas player $i$ 's deviation to $\rho=1$ in any period leads immediately to the CC-game whose equilibrium is described in case (i). There exists no PBE in pure participation strategies exists if $\pi_{i}^{1}>\bar{\pi}(\delta)$ for $i=A, B$.

Proof. See the Appendix.

Thus, the CC-game is reached immediately with probability one in the following two cases. (i) The discount factor is high $\left(\delta \geq \delta_{c}\right)$, (ii) the discount factor is low $\left(\delta<\delta_{c}\right)$ but both prior beliefs do not exceed $\bar{\pi}(\delta)$, a critical level associated with $\delta$. In these two cases, the inclusion of incomplete information has no impact on the pattern of contributions, hence a low discount factor is necessary but not sufficient for a pure defection advantage to arise. Very asymmetric prior beliefs coupled with low discount factors may lead to the $\mathrm{CN}$ - or NC-game where the pessimistic player concedes immediately. Though a perpetual defection is individually the first-best outcome, the pessimistic player gives in and contributes $Q^{\mathrm{HL}}$ forever. This represents the third type of inefficiency in the private supply of public goods mentioned in the Introduction: Total per-period contributions in the $\mathrm{CN}$ or NC-game $\left(Q^{\mathrm{HL}}\right)$ are even lower than $\left(2 Q^{\mathrm{HH}}\right)$, total contributions in the static play of the complete information stage game. If both players are impatient but none is pessimistic in the sense described above, another interesting outcome arises. In fact, Proposition 4 below shows that if future payoffs are heavily discounted, increasing the players' optimism intensifies the free-riding problem even in a repeated game setting.

Proposition 4. Assume $\delta<\delta_{c}$ and $\bar{\pi}(\delta) \leq \pi_{i}^{1}<1$ for $i=A, B$. There exists a unique undominated $P B E$ path, initially in completely mixed participation strategies $\left(\left\{\rho_{A}^{t *}\right\}\right.$, $\left.\left\{\rho_{B}^{t *}\right\}\right)$, and a finite integer $T>1$ such that a contribution occurs at date $T$ with probability one.

Proof. See the Appendix.

The equilibrium outcome of the repeated game may involve delayed contributions under the conditions given in Proposition 4. The corresponding equilibrium path has the property that the players hesitate between participation and defection; they revise their beliefs and put more weight on participation if no contribution has 
been observed. Eventually these beliefs fall below the critical level $\bar{\pi}(\delta)$ and a pure participation strategy is played. Of course, contributions may start earlier as a result of mixing. In each period along this equilibrium path, the expected payoff from participation equals the expected payoff from pure defection, given the other player's random participation strategy.

The equilibrium in completely mixed participation strategies is generated by the potential pure defection advantage. The gains from a unilateral defection are quite attractive but uncertain. Note that the outcome changes dramatically if one of the players becomes the informed player; now this player knows the gains from defecting forever, which, if $\bar{P}(\delta)<U\left(Q^{\mathrm{HL}}\right)$, are larger than those he obtains by participating and revealing his type. The overall game then turns into the $\mathrm{CN}$ - or NC-game where participation strategies are pure, and the player identified as type- $H$ has no means to avoid the burden of providing the public good. His threat to defect is not credible because the informed player's type cannot be verified. Also in the overall game played under two-sided incomplete information, unilateral free-riding threats or commitments at the beginning of the game are not credible and are eliminated by the PBE concept.

The predictions of the model can be reformulated in light of the interpretations of the discount factor mentioned in the beginning of this section. If the players are interacting frequently or if the probability that the game ends is low, introducing incomplete valuation information has no effect on the pattern of contributions. It is only when the length of a period is sufficiently high or the frequency of interaction is low that the outcome depends critically on the distribution of prior beliefs.

\section{Conclusions}

This paper studies the impact of incomplete information on the pattern of contributions and incentives to free ride. It shows that contrasting patterns of contributions may arise as a function of the discount factor, initial distribution of beliefs and the level of expected payoffs in the potential complete information subgame. The free-riding problem is not necessarily aggravated by inefficient delays or very low contributions as in Bliss and Nalebuff (1984) or Gradstein (1992); the opposite happens for a wide range of discount factors. This difference in results stems from the differences in assumptions, discussed in Section 2. The two papers mentioned above assume fixed contributions and a finite number of periods (one and two, respectively), which means that the players will never face a similar public good provision problem in the future. This is quite restrictive considering the recurrent interaction of individuals in many social settings; it greatly reduces the potential for cooperation in the spirit of the chain-store paradox. In a repeated game with continuous contributions, we show that if the discount factor is not too low, expected payoffs in the potential complete information subgame are sufficiently high and thus the inclusion of incomplete information has no effect on the pattern of contributions. This outcome can also arise when the discount factor is low but all players hold pessimistic prior beliefs. Given a low discount factor, the overall game may collapse into a war of attrition if players become increasingly optimistic. In a war of attrition, the players' reluctance to participate and reveal their valuations generates delay with positive probability. The conceding player supplies the public good in all future periods whereas the other defects forever. 


\section{Appendix}

Proof of proposition 3. Notice that any undominated CC-, NC- or CC-outcome that occurs through the play of pure participation strategies must occur in period one. Hence, ruling out dominated equilibria, delay may occur only through the play of mixed strategies. The proofs of cases (i) and (ii) consist of deriving the best reply functions and then using these functions to construct the PBE presented in the Proposition.

Case (i). Consider $A$ 's best reply to $\left\{\rho_{B}^{1}\right\}=\{1,1, \ldots\} . A$ has two choices in pure strategies in the first period: The first is to play $\rho_{A}^{1}=1$. If $B$ too reveals that he is of type- $H$, we are in the CC-game where average symmetric payoffs are $\bar{P}(\delta)$. Second, player $A$ can also choose to mimic type- $L\left(\rho_{A}^{1}=0\right)$ in period one, and then reveal his type or not in the second period after observing $B$ 's strategy. That is, $A$ can play $\left\{\rho_{A}^{2}\right\}=\{0,0, \ldots\}$ if $B$ turns out to be of type- $H$, and $\left\{\rho_{A}^{2}\right\}=\{1,1, \ldots\}$ otherwise. This strategy is the best alternative to the first. Comparing these two pure strategy options yields

$$
\pi_{B}^{1}\left(\bar{P}(\delta)-U\left(Q^{\mathrm{HL}}\right)\right)+\left(1-\pi_{B}^{1}\right)(1-\delta)\left[U\left(Q^{\mathrm{HL}}\right)-C\left(Q^{\mathrm{HL}}\right)\right] \geq 0
$$

which states the condition under which $A$ should prefer the first option, and thus $\left\{\rho_{i}^{1}\right\}=\{1,1, \ldots\}$ for $i=A, B$ form a PBE. Clearly, (8) holds no matter the value of $\pi_{B}^{1}$ if $\delta \geq \delta_{c}$, which implies the result in case (i) by symmetry.

Case (ii). Consider now the case $\delta<\delta_{\mathrm{c}}$. Since $\bar{P}(\delta)-U\left(Q^{\mathrm{HL}}\right)<0$, the left hand side of $(8)$ is decreasing in $\pi_{B}^{1}$. Define the schedule $\bar{\pi}(\delta)$ by the function $\bar{\pi}:\left[0, \delta_{c}\right] \rightarrow[0,1]$, such that (8) holds with equality if $\pi_{B}^{1}=\bar{\pi}(\delta)$. Given $\delta<\delta_{c}$, we obtain the equilibrium in case (i) if $\pi_{i}^{1} \leq \bar{\pi}(\delta)$. Inspection of (8) reveals that for the case of asymmetric priors, $\pi_{i}^{1} \leq \bar{\pi}(\delta)<\pi_{j}^{1}$, the strategies described in the proposition are mutually best replies. (8) holds for player $j$ who therefore chooses $\left\{\rho_{j}^{1 *}\right\}=\{1,1, \ldots\}$ no matter the strategy of player $i$. Given this strategy of $j$, the best reply of $i$ is to set $\left\{\rho_{i}^{1 *}\right\}=\{0,0, \ldots\}$ because $\bar{\pi}(\delta)<\pi_{j}^{1}$. The postulated sequences of beliefs are consistent with the strategies, and no player has any incentive to deviate given what happens off the equilibrium path. Finally, the non-existence of a pure concession strategy equilibrium if $\bar{\pi}(\delta)<\pi_{i}^{1}$ for $i=A, B$ can be verified by making use of (8) and Proposition 2. Q.E.D.

Proof of proposition 4. As stated in Proposition 2, "no contribution ever occurs" is not a pure strategy PBE outcome. It is also easy to show that such an outcome cannot happen if we allow for mixed strategies. Along any arbitrary pattern of mixed strategies, revision of beliefs according to Bayes' rule will generate a declining pattern of beliefs, which will eventually fall below the critical level $\bar{\pi}(\delta)$ and a pure participation strategy will be played. Hence, a finite date $T$ exists where at least one player contributes. Now assume $\bar{\pi}(\delta) \leq \pi_{i}^{1}<1$ for $i=A, B$. As mentioned, if the first contribution occurs at date $T$, at least one player's belief must be less than or equal to $\bar{\pi}(\delta)$. In this proof we consider the case $\pi_{A}^{T}<\bar{\pi}(\delta) \leq \pi_{B}^{T}$ where by Proposition 3 (ii) $A$ 's optimal strategy in period $T$ is $\rho_{A}^{T *}=0$ and $B$ 's is $\rho_{B}^{T *}=1$ (the proofs for the cases $\pi_{B}^{T}<\bar{\pi}(\delta) \leq \pi_{A}^{T}$ and $\pi_{i}^{T}<\bar{\pi}(\delta)$ use similar arguments.)

Claim 1. $0<\rho_{i}^{T-1^{*}}<1$.

Proof. If $\rho_{i}^{T-1}=1$, then the public good would be provided at date $T-1$, which contradicts our assumption. On the other hand, if $\rho_{A}^{T-1}=0$, then $\pi_{A}^{T-1}=\pi_{A}^{T}$. But since $\pi_{A}^{T}<\bar{\pi}(\delta)$, the optimal strategy of $B$ would be $\rho_{B}^{T-1}=1$, leading to 
a contribution at date $T-1$, a contradiction. Therefore, $0<\rho_{A}^{T-1^{*}}<1$. The proof of $0<\rho_{B}^{T-1^{*}}<1$ proceeds along the same lines. Q.E.D.

Claim 2. $\pi_{A}^{T}<\bar{\pi}(\delta) \leq \pi_{A}^{T-1}$.

Proof. $0<\rho_{A}^{T-1^{*}}<1$ implies $\pi_{A}^{T}<\pi_{A}^{T-1}$ through Bayes' rule. Finally, if $\pi_{A}^{T-1}<\bar{\pi}(\delta)$, then $\rho_{B}^{T-1}=1$, which contradicts Claim 1. Q.E.D.

At the beginning of period $T$ (before participation strategies are played), the players' normalized expected payoffs are respectively given by

$$
(1-\delta) W_{A}^{T}=\pi_{B}^{T} U\left(Q^{\mathrm{HL}}\right)+\left(1-\pi_{B}^{T}\right) \delta P_{L} \text { and }(1-\delta) W_{B}^{T}=P_{L},
$$

where $P_{L}=U\left(Q^{\mathrm{HL}}\right)-C\left(Q^{\mathrm{HL}}\right)$. Let us now move backwards to period $T-1$. By Claims 1 and 2 we must have $\pi_{i}^{T-1}>\bar{\pi}(\delta)$ holding for both players, which in turn implies, by Proposition 3, that the equilibrium strategies in period $T-1$ cannot be pure. We construct an equilibrium in completely mixed participation strategies below. The normalized expected payoffs in period $T-1$ associated with pure participation and pure defection strategies are respectively as follows: Playing $\rho_{B}^{T-1}=1$ yields

$$
\pi_{A}^{T-1} \rho_{A}^{T-1} \bar{P}(\delta)+\left(1-\pi_{A}^{T-1} \rho_{A}^{T-1}\right) P_{L}
$$

while $\rho_{B}^{T-1}=0$ yields

$$
\pi_{A}^{T-1} \rho_{A}^{T-1} U\left(Q^{\mathrm{HL}}\right)+\left(1-\pi_{A}^{T-1} \rho_{A}^{T-1}\right) \delta P_{L},
$$

given the optimal strategies $\rho_{A}^{T^{*}}=0$ and $\rho_{B}^{T^{*}}=1$ for the next period. Similarly,

$$
\pi_{B}^{T-1} \rho_{B}^{T-1} \bar{P}(\delta)+\left(1-\pi_{B}^{T-1} \rho_{B}^{T-1}\right) P_{L},
$$

is the payoff player $A$ expects from $\rho_{A}^{T-1}=1$, and using (9),

$$
\pi_{B}^{T-1} \rho_{B}^{T-1} U\left(Q^{\mathrm{HL}}\right)+\left(1-\pi_{B}^{T-1} \rho_{B}^{T-1}\right) \delta\left[\pi_{B}^{T} U\left(Q^{\mathrm{HL}}\right)+\left(1-\pi_{B}^{T}\right) \delta P_{L}\right]
$$

from playing $\rho_{A}^{T-1}=0$.

Claim 3. $\delta\left[\pi_{B}^{T} U\left(Q^{\mathrm{HL}}\right)+\left(1-\pi_{B}^{T}\right) \delta P_{L}\right]<P_{L}$.

Proof. Assume the contrary. $\delta<\delta_{c}$ implies $\widetilde{P}(\delta)<U\left(Q^{\mathrm{HL}}\right)$, and from (12) and (13) the optimum strategy for $A$ is $\rho_{A}^{T-1}=0$. But since $\rho_{A}^{T^{*}}=0$ too, player $B$ would be better-off setting $\rho_{B}^{T-1}=1$ (participating in period $T-1$ ) which contradicts Claim 1. Q.E.D.

Given the result in Claim 3 and $\pi_{i}^{T-1}>\bar{\pi}(\delta)$ for $i=A, B$, there must exist numbers $0<\rho_{i}^{T-1^{*}}<1$ such that

$$
\rho_{A}^{T-1^{*}} \pi_{A}^{T-1}=\frac{1}{1+\left[U\left(Q^{\mathrm{HL}}\right)-\bar{P}(\delta)\right] /(1-\delta) P_{L}}
$$

and

$$
\rho_{B}^{T-1^{*}} \pi_{B}^{T-1}=\frac{1}{1+\left[U\left(Q^{\mathrm{HL}}\right)-\bar{P}(\delta)\right] /\left[P_{L}-\delta\left(\pi_{B}^{T} U\left(Q^{\mathrm{HL}}\right)+\left(1-\pi_{B}^{T}\right) \delta P_{L}\right)\right]} .
$$

The strategies above are determined respectively using (10), (11), (12) and (13), and are unique. Thus the respective payoffs of the players, discounted to $T-1$ are given by $W_{A}^{T-1}=\rho_{A}^{T-1^{*}}(12)+\left(1-\rho_{A}^{T-1^{*}}\right)(13)$ and $W_{B}^{T-1}=\rho_{B}^{T-1^{*}}$ $(10)+\left(1-\rho_{B}^{T-1^{*}}\right)(11)$. Clearly, $\rho_{A}^{T-1^{*}}$ and $\rho_{B}^{T-1^{*}}$ are best replies to each other and beliefs are consistent with these strategies. We have thus far constructed the portion 
of the PBE path for periods $T$ and $T-1$ under the assumption that the NC-game is reached at $T$ with probability one. Given $W_{A}^{T-1}$ and $W_{B}^{T-1}$, we can proceed to period $T-2$ and determine the optimal (mixed) participation strategies, using Bayes' rule for updating beliefs as we did for period $T-1$. Carrying this process inductively until period one should lead to the initial conditions described by $\pi_{i}^{1}>\bar{\pi}(\delta)$ and $\delta<\delta_{c}$. The portion of the overall game ending at date $T$ can be viewed as a finite game if we exclude the contribution games in each period. Thus the number of equilibria of this game must be odd. Since the completely mixed participation strategies corresponding to the initial conditions described in the proposition are uniquely determined, and since no equilibrium in pure participation strategies exists, this number must be equal to one. Q.E.D.

Acknowledgement. I wish to thank two anonymous referees for helpful comments and suggestions. Remaining errors are mine.

\section{References}

Admati A, Perry M (1991) Joint projects without commitment. Rev Econ Studies 58: $259-276$

Andreoni J (1988) Privately provided public goods in a large economy: the limits of altruism. J Public Econ 35: 57--73

Bergstrom T, Blume L, Varian H (1986) On the private provision of public goods. J Public Econ 29: 25-49

Bliss C, Nalebuff B (1984) Dragon slaying and ballroom dancing: the private supply of a public good. J Public Econ 25: 1-12

Bulow J, Geanakoplos J, Klemperer P (1985) Multimarket oligopoly: strategic substitutes and complements. J Polit Econ 93: 488-511

Chatterjee K, Samuelson L (1987) Bargaining with two-sided incomplete information: an infinite horizon model with alternating offers. Rev Econ Studies 54: 175-192

Fershtman C, Nitzan S (1991) Dynamic voluntary provision of public goods. European Econ Rev 35: 1057-1067

Fudenberg D, Tirole J (1984) The fat cat effect, the puppy dog ploy and the lean and hungry look. Amer Econ Rev Papers and Proceedings 74: 361-368

Fudenberg D, Tirole J (1986) A theory of exit in oligopoly. Econometrica 54: 943-960

Gradstein M (1992) Time dynamics and incomplete information in the private provision of public goods. J Polit Econ 100: 581-597

Kreps D, Wilson RB (1982) Reputation and imperfect information. J Econ Theory 27: 253-279

McMillan J (1979) Individual incentives in the supply of public inputs. J Public Econ 12: $87-98$

Nalebuff B, Riley J (1985) Asymmetric equilibria in the war of attrition. $J$ Theoretical Biology 113: 517-528

Palfrey TR, Rosenthal $H$ (1984) Participation and provision of discrete public goods: a strategic analysis. J Public Econ 24: 171-193

Palfrey TR, Rosenthal H (1988) Private incentives in social dilemmas. J Public Econ 35: $309-332$

Radner R (1986) Repeated partnership games with imperfect information and no discounting. Rev Econ Studies 53: 43-58

Roth A (1987) Bargaining phenomena and bargaining theory. In: Roth A (ed) Laboratory Experimentation in Economics: Six Points of View. New York, Cambridge University Press

Schelling T (1966) Arms and influence. New Haven, CT, Yale University Press 